\title{
DNA barcodes from four loci provides poor resolution on phylogenetic relationships between the Triticum species
}

\author{
Kim Seong-Hoon ${ }^{1}$, Raveendar Sebastin ${ }^{1}$, Hyun Do Yoon ${ }^{1}$, Lee Gi-An², \\ Xiaohan Wang ${ }^{3}$, Lee Kyung Jun ${ }^{1}$, Shin Myoung-Jae ${ }^{1}$, Lee Jung-Ro ${ }^{1}$, \\ Lee Sookyeong ${ }^{1}$, Han Sea-hee ${ }^{4}$, Cho Gyu-Taek ${ }^{1}$ \\ ${ }^{1}$ National Institute of Agricultural Sciences, RDA, Republic of Korea \\ ${ }^{2} R \& D$ Coordination Division, $R D A$, Republic of Korea \\ ${ }^{3}$ Kyungpook National University, Republic of Korea \\ ${ }^{4}$ Chungbuk National University, Republic of Korea \\ *e-mail: shkim0819@korea.kr
}

DNA barcoding relatively a novel approach, which was developed to provide rapid, accurate and automatable species identification by using standardized DNA regions. The Consortium for the Barcode of Life (CBOL) plant-working group recommended the 2-locus combination as the standard plant barcode. The evolutions of the chloroplast regions combine with nuclear gens are sufficiently rapid to allow discrimination between closely related species. In this study, we tested the phylogenetic utility of the DNA barcoding loci (ITS2, matK, psbA-trnH and $r b c L$ ) for efficient discrimination of Triticum species. To assess the barcoding efficiency to resolve the species discrimination, a total of 109 accessions representing 16 recognized genotypes in the Triticum genus have been sampled. Topologies of the phylogenetic trees based on combination of DNA barcode analyses were similar, but a few accessions were placed into distant phylogenetic groups. The 109 accessions analyzed in this study were placed into three groups supported by high bootstrap values. However, as expected the barcoding analyses were not able to discriminate some closely related Triticum species. Thus, we have proposed, molecular studies with more diverse markers and species will be required to clarify the ambiguities surrounding the phylogeny of these important genera. 